\title{
Binding of colicins A and E1 to purified TolA domains
}

\author{
Rahmona Derouiche, ${ }^{1}$ Gabrielle Zeder-Lutz, ${ }^{2}$ Hélène Bénédetti, ${ }^{1}$ \\ Marthe Gavioli, ${ }^{1}$ Alain Rigal, ${ }^{1}$ Claude Lazdunski' and Roland Lloubès ${ }^{1}$
}

Author for correspondence: Roland Lloubès. Tel: +3349112645 54. Fax: +33491712124. e-mail: lloubes@ibsm.cnrs-mrs.fr

\footnotetext{
1 Laboratoire d'Ingénierie et Dynamique des Systèmes Membranaires, UPR 9027, Institut de Biologie Structurale et Microbiologie, 13402 Marseille Cedex 20, France

2 Laboratoire d'Immunochimie des Peptides et Virus, UPR 9021, 67084 Strasbourg Cedex, France
}

\begin{abstract}
Colicins are divided into two groups according to the proteins required for their import into sensitive bacteria. The Tol and TonB pathways are involved in import of group A and group B colicins respectively. Because previous analyses have shown that colicin E1 and colicin A (two group A colicins) interact in vitro with the C-terminal domain of TolA (TolAIII) while colicin B (group B colicin) does not, attention was focused on these interactions with purified proteins. TolA has been described as a three-domain protein with an $\mathbf{N}$-terminal innermembrane anchor and a long periplasmic region formed by two domains (ToIAII and ToIAIII). ToIAIII, ToIAII and ToIAll-III soluble domains with an Nterminal hexa-histidine extension were purified. The interactions of colicins with the purified TolA domains were analysed by overlay Western blotting, which indicated that both $\mathbf{N}$-terminal domains of colicins A and E1 interacted with TolAIII, while a gel shift procedure detected no interaction with colicin E1. The binding kinetic values of the N-terminal domains of colicins A and E1 to TolAlll were estimated by surface plasmon resonance and were shown to be similar.
\end{abstract}

Keywords: Tol proteins, colicins, protein interactions

\section{INTRODUCTION}

The Tol and TonB systems are involved in macromolecule import into Escherichia coli. Colicins and phage DNA have parasitized these protein complexes to penetrate into bacterial cells. In order to reach their cellular targets, colicins follow a three-step process. First, they bind to bacterial receptors; second, they translocate through the outer membrane; and third, they act either by forming a pore into the inner membrane or by exerting nuclease activity in the cell cytoplasm (for reviews see James et al., 1996; Lazdunski, 1995). The TonB system is required for active transport of vitamin $B_{12}$ and siderophores (Postle, 1993), while the function of the Tol system is not yet understood, although it is required for the integrity of the cell envelope (Webster, 1991; Fognini-Lefébvre et al., 1987; Lazzaroni \& Portalier, 1992). The TolA/B/Q/R/Pal and Orf2 proteins form the Tol system. The topologies of the inner-membrane-anchored TolA, TolQ and TolR proteins have been determined (Levengood \& Webster,

Abbreviation: SPR, surface plasmon resonance.
1989; Kampfenkel \& Braun, 1993; Vianney et al., 1994; Müller et al., 1993). TolB and Orf2 have been found to be periplasmic proteins (Isnard et al., 1994; Vianney et al., 1996). TolA, TolQ and TolR form an innermembrane Tol-protein complex, with the membrane domain of TolA interacting with TolQ and TolR (Derouiche et al., 1995), while the membrane anchor of TolR was shown to interact with the third membrane segment of TolQ (Lazzaroni et al., 1995). An outermembrane protein complex consisting of the periplasmic TolB protein and the lipoprotein Pal was also found (Bouveret et al., 1995). Furthermore, cell-fractionation experiments have revealed that TolQ, $-\mathrm{R},-\mathrm{A}$ and $-\mathrm{B}$ form a protein complex of defined stoichiometry (Guihard et al., 1994). TolA has been described as a three-domain protein (Levengood et al., 1991), corresponding to a membrane anchor, a central domain and a C-terminal domain (domains I, II and III, respectively). In vitro, the TolA central domain (domain II) interacts with outer-membrane trimeric porins and its function has been suggested to be related to the assembly process of outer-membrane porins requiring LPS (Derouiche et al., 1996). When expressed and exported to the periplasm of wild-type cells, TolA 
domain III causes the same phenotypic effects as a tol mutation; moreover, domain III alone competes with TolA for group A colicin import (Levengood-Freyermuth et al., 1993). We previously demonstrated, using crude cell extracts, that TolAIII interacted with colicins and that these interactions might be relevant to a step in the colicin import process through the outer membrane (Bénédetti et al., 1991). Recently, TolB was shown to interact with the colicin E3 N-terminal domain and, thus, might also play a role in the translocation step of colicins (Bouveret et al., 1997). In this paper, the interactions of purified TolA periplasmic domains with two pore-forming colicins and their translocation domain were analysed by using overlay Western blotting, gel shift assays and surface plasmon resonance (SPR) techniques.

\section{METHODS}

Plasmid constructs. Plasmid pAR3 (Perez-Perez \& Gutierrez, 1995) contains the chloramphenicol-resistance gene and ori from pACY184 with the $\operatorname{araC}$ gene and the $a r a B$ promoter followed by the multiple cloning site from pAra12 (Cagnon $e t$ al., 1991). An additional transcriptional terminator formed by an 81 bp DNA fragment from the crystal protein of Bacillus thuringiensis was inserted into the BglII restriction site, giving plasmid pART3 (J. Gutierrez, unpublished).

First, we inserted into the unique PstI site of pART3 a synthetic DNA fragment (5'-CATCATCATCATCATCACC GCATGCAAGCGGCCGCAAGGCCT-3') encoding six histidines, giving vector pARTHis. The six histidine codons are followed by three unique restriction sites, SphI, NotI (or EagI) and StuI (underlined).

The plasmids encoding the TolA domain II-III and domain III were obtained by inserting the 1146 bp SpbI-HpaI and the 447 bp EagI-HpaI DNA fragments of pTPS306 (Sun \& Webster, 1986) into pARTHis digested by SphI-StuI and EagI-StuI, respectively. The plasmid encoding TolAII was obtained in two steps. First, the unique EspI site of pART3 was destroyed after being filled with Klenow polymerase and ligated. The SphI-HpaI DNA fragment encoding TolAII-III was inserted into this plasmid. Then, a synthetic DNA fragment containing two stop codons ( $5^{\prime}$-TAAGAGATCTGTAATGAACCATGGAA-3') was inserted into the unique EspI site of the tolAII-III gene. These three plasmids were called pARTolAII-III, pARTolAIII and pARTolAII.

Plasmid $\mathrm{pHS}$ was obtained from plasmid $\mathrm{pBRE} 1 \mathrm{SX}$ in which two restriction sites were introduced between the three domains of the colicin E1 gene (Frenette et al., 1991). The SpeI-AflI DNA fragment was deleted and thus plasmid pHS encodes the N-terminal domain of colicin E1 (HS domain).

Purification of TolA derivatives and $\mathrm{N}$-terminal domains of colicins A and E1. The plasmids encoding TolAII-III and TolAIII were transformed into the recipient MC1061 cells (Cagnon et al., 1991). TolAII, which was poorly induced in MC1061 (this might be due to the EspI mutation in the araC gene), was expressed in C600 cells. Briefly, cultures were grown in LB (containing $30 \mu \mathrm{g}$ chloramphenicol $\mathrm{ml}^{-1}$ ) to $\mathrm{OD}_{600} 1 \cdot 0$. TolA expression was induced with $0.5 \mathrm{mg}$ arabinose $\mathrm{ml}^{-1}$ for $2 \mathrm{~h}$. Cells were collected by centrifugation, lysed and further sonicated. After centrifugation at $100000 \mathrm{~g}$, supernatants were directly incubated with nickel or cobalt beads $(\mathrm{Nb}$ or $\mathrm{Cb}$, respectively) for $1 \mathrm{~h}$. All buffers used for purification with $\mathrm{Nb}(50 \mathrm{mM}$ sodium phosphate $\mathrm{pH} 8.0$, $300 \mathrm{mM} \mathrm{NaCl}, 0.5 \mathrm{mM}$ PMSF) and $\mathrm{Cb}(20 \mathrm{mM}$ Tris/HCl pH $8.0,100 \mathrm{mM} \mathrm{NaCl}, 0.5 \mathrm{mM}$ PMSF) are described by Qiagen (Quiaexpressionist) and Clontech (TALON metal affinity resin), respectively. After washing, the TolA derivatives were eluted with between 50 and $150 \mathrm{mM}$ imidazole

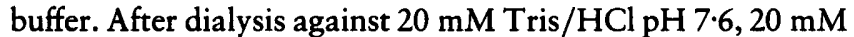
$\mathrm{NaCl}$ buffer, the second step of purification was carried out using MonoS cation-exchange chromatography (Pharmacia). TolAII-III and TolAIII were eluted with between 0.1 and $0.3 \mathrm{M} \mathrm{NaCl}$; cation-exchange chromatography was not performed for TolAII.

AT1 and HS (N-terminal domains of colicins $\mathrm{A}$ and $\mathrm{E} 1$, respectively) were purified from $\mathrm{C} 600$ cell supernatants after induction with mitomycin C. Briefly, after ammonium sulphate precipitation and dialysis against $50 \mathrm{mM}$ phosphate buffer $\mathrm{pH} \mathrm{6.8,} \mathrm{the} \mathrm{N}$-terminal domains were purified by MonoS chromatography as described for AT1 by Knibiehler et al. (1989). Colicin A was purified as described by Baty $e t$ al. (1987), while colicin E1 was a gift from W. A. Cramer (Purdue University, Ind., USA).

Amino acid compositions and $\mathbf{N}$-terminal protein sequences. Amino acid composition determinations performed on purified TolA derivatives, AT1 and HS proteins gave the expected ratios. From two amino acid compositions, the $\varepsilon_{280}$ values of TolAII-III and TolAIII were calculated to be 10300 and $6900 \mathrm{M}^{-1} \mathrm{~cm}^{-1}$, respectively. N-terminal sequences of TolAIII and TolAII-III were checked and the results indicated that the $\mathrm{N}$-terminal formylmethionine was removed. Since TolAII does not have many aromatic residues (no W, no Y and only one $\mathrm{F}$ residue), the $\varepsilon_{280}$ was not estimated.

Western blot analysis. Overlay techniques have been previously described by Bénédetti et al. (1991). Briefly, TolA derivatives were heat-denatured with reducing agent in sample buffer before SDS-PAGE. After Western blotting of purified TolA derivatives, colicins were first incubated and immunodetected with their respective antibodies: monoclonal antibodies (mAbs) 1C11 or 3D6 for colicin A (Cavard et al., 1986), or the polyclonal antibody ( $\mathrm{pAb}$ ) raised against the $\mathrm{N}$-terminal domain of colicin E1 (anti-HS antibody), or a pAb raised against colicin E1 or colicin B. Then, immunodetection of colicin at the corresponding migration mobility of the TolA derivative confirmed that colicin interacted with purified TolA domains. The antibodies were previously checked for the absence of cross-reactivity with TolA.

SDSPAGE shift assay. Protein complexes formed between TolA derivatives and either colicin A (or AT1), or colicin E1 (or HS) were analysed by SDS-PAGE. The incubations ( 15 to $30 \mathrm{~min}$ ) of TolA with colicin derivatives were carried out in $10 \mathrm{mM}$ Tris/ $\mathrm{HCl} \mathrm{pH} \mathrm{8.0,100} \mathrm{mM} \mathrm{NaCl}$. Samples were directly applied with the addition of sample buffer (devoid of SDS, dye and reducing agent) on SDS-PAGE without any denaturing step and the electrophoreses run between 10 and $20 \mathrm{~V} \mathrm{~cm}^{-1}$. Purified proteins were analysed by Coomassie blue staining.

Peptide synthesis. Two peptides encompassing the putative N-terminal 'TolA box' sequence (Pilsl \& Braun, 1995) were synthesized and further purified by reverse-phase chromatography to perform competition-binding to TolAIII with their respective $\mathrm{N}$-terminal domains. The sequences correspond to $\mathrm{NH}_{2}-\mathrm{G}_{27}$ TPDGSGSGGG-COOH (G11G) and $\mathrm{NH}_{2}-\mathrm{G}_{8} \mathrm{~K}-$ GDGTGWSSE-COOH (G11E). The putative TolA boxes are underlined, with the position of the first residue of colicin E1 and colicin A, respectively, indicated. 
SPR (BIAcore). The BIAcore system and reagents for interaction analysis including CM5 sensor chips, surfactant P20, $N$-hydroxysuccinimide (NHS) and $N$-ethyl- $N^{\prime}$-(3-dimethylaminopropyl)carbodiimide hydrochloride (EDC) were obtained from Pharmacia Biosensor. Interaction analysis was performed in $10 \mathrm{mM}$ HEPES, $150 \mathrm{mM} \mathrm{NaCl}, 3.4 \mathrm{mM}$ EDTA, $0.005 \%$ surfactant P20, pH 7.4 (HBS buffer).

TolA derivatives diluted (about 10 -fold) to $100 \mu \mathrm{M}$ in $10 \mathrm{mM}$ acetate pH 5.0 were immobilized on CM5 sensor chips through primary amino groups according to the standard EDC/NHS procedure (Johnsson \& Löfås, 1991). Concentrations ranging from 0.2 to $2 \mu \mathrm{M}$ of colicins A, AT1, colicin E1 and HS in HBS buffer were used. To suppress the interaction observed when colicins A and E1 were injected onto the dextran matrix of the sensor chip, the colicins were diluted in HBS with $1 \mathrm{mg}$ carboxymethyldextran $\mathrm{ml}^{-1}$ (Fluka). Colicin B was used as a negative control. Regeneration steps were optimized for each protein to recover at least $90 \%$ of the initial binding after many (10 to 20$)$ cycles. Reciprocal interactions with immobilized colicins $\mathrm{A}$ and E1 and TolA derivatives injected into the buffer flow were also analysed. Experimental data were obtained in the form of sensorgrams, which show the amount of interacting molecules (expressed as resonance units, $\mathrm{RU}$ ) as a function of time. A signal of 1000 RU corresponds to fixation of $1 \mathrm{ng}$ protein $\mathrm{mm}^{-2}$.

Kinetic data were interpreted with BIAevaluation 2.1 software. The association $\left(k_{\mathrm{a}}\right)$ and the dissociation $\left(k_{\mathrm{d}}\right)$ rate constants were calculated from the injection and post-injection phases of the interaction curve by a nonlinear regression method (O’Shannessy et al., 1993).

\section{RESULTS}

\section{Plasmid constructs and protein purification}

Large amounts of TolA derivatives were obtained using the tightly regulated $a r a B$ promoter from pART3 (see Methods). This plasmid contained the ara $C$ gene encoding the arabinose repressor acting on the $a r a B$ promoter. To facilitate protein purification, a synthetic DNA fragment encoding an N-terminal six-histidine tagging sequence was added. Three plasmids, pARTolAII, pARTolAIII and pARTolAII-III, were constructed which expressed TolAII, TolAIII and TolAII-III proteins corresponding to 236 residues of the central domain, 132 residues of the C-terminal domain and 355 residues of the two soluble domains, respectively (Fig. 1). Soluble TolA derivatives were overproduced after $2 \mathrm{~h}$ of arabinose induction and were recovered after sonication of the cell pellet and ultracentrifugation. The first step of purification was obtained by chelating the histidine-tagged recombinant proteins on NTAnickel beads $(\mathrm{Nb})$ and eluting them by a batch procedure using increasing concentrations of imidazole buffer. By this approach, in addition to TolA derivatives, a protein was eluted between 20 and $40 \mathrm{mM}$ imidazole. The $\mathrm{N}-$ terminal sequence of this protein was determined after being transferred onto a PVDF membrane. The first 10 residues, MTIFDNYEVN, were found to correspond to E. coli arabinose isomerase (which contains 21 histidines among the 500 residues). Cationic exchange chromatography was performed for TolAIII and TolAII-III purification to eliminate contaminants. TolA deri- vatives, colicins $\mathrm{A}$ and $\mathrm{E} 1$ and their $\mathrm{N}$-terminal domains are shown in Fig. 2 and represent at least $90 \%$ purified proteins.

\section{Overlay immunodetection of colicin binding to Western-blotted TolA derivatives}

The antibodies were checked for their cross-reactivity with TolA derivatives before overlay immunodetection. A low background interaction was observed using the anti-HS antibody due to cross-reaction with TolA domain II; all the other antibodies, including the anticolicin E1 antibody, did not cross-react with TolA. The overlay assays were performed according to the immunodetection of bound colicin on Western-blotted TolA domains. By this approach using anti-colicin E1 and anti-HS antibodies colicin E1 was clearly detected on Western-blotted TolAIII while its $\mathrm{N}$-terminal domain (HS) was poorly immunodetected. To avoid any steric hindrance that might occur between the antibody recognition sequence and the region interacting with TolAIII, we used the hybrid colicin E1AA, which comprises the $\mathrm{N}$-terminal domain of colicin $\mathrm{E} 1$ (identical to that of the HS protein) and the central and C-terminal domain of colicin A (Frenette et al., 1991). Its binding to TolA was immunodetected by using mAb 3D6 (Cavard et al., 1986) (a mAb directed against an epitope previously shown to contain residues 372 to 388 of colicin A; Bénédetti et al., 1991). This experiment demonstrated that the N-terminal domain of colicin E1 was able to interact with the C-terminal domain of TolA because the two colicin A domains present in E1AA did not interact with TolA (as previously shown by Bénédetti et al., 1991). In conclusion, these results indicated that the N-terminal domain of colicins $\mathrm{A}$ and E1 interacted with TolAIII but not with TolAII (Table 1).

\section{Gel shift assays of colicins binding to TolA}

When colicin A and AT1 were incubated with TolAII-III or TolAIII without denaturing agent and further analysed by SDS-PAGE shift assay, high-molecular-mass bands appeared after Coomassie blue staining (Fig. 3). Western blot immunodetections indicated that these bands corresponded to complexes formed by TolA and colicin A (not shown). According to the molecular masses of these complexes, they might correspond to a heterodimer formed by colicin A (or AT1) and TolAIII (or TolAII-III). Upon heat denaturation the monomeric form of each protein was recovered. No protein complex was observed between TolAII and colicin A, and no TolA derivative interacted with colicin E1 and HS to form a band shift under the same conditions (Fig. 3). As a negative control, when colicin B or the C-terminal domain of colicin A was used, no complex was detected (not shown).

\section{Estimation of the kinetic values of colicin binding to immobilized ToIAIII by SPR measurements}

The biosensor monitors interactions between pairs of molecules when one partner (the ligand) is immobilized 

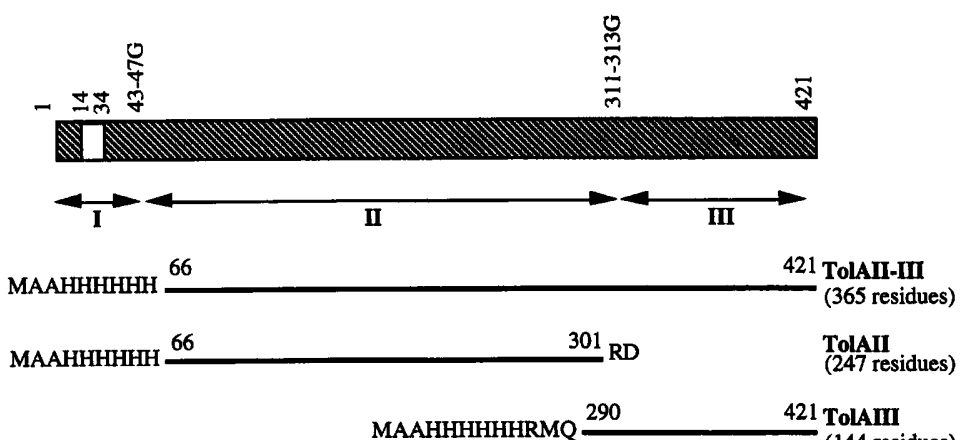

MAAHHHHHHRMQ $\frac{290 \quad 421}{\text { TolAIII }}$ (144 residues)
Fig. 1. Amino acid sequences of TolA derivatives. The two glycine clusters corresponding to the domain boundaries of TolA are indicated (residues 43 to 47 and 311 to 313 ). The inner-membrane-spanning segment is indicated by a white box. The $\mathrm{N}$-terminal-tagged TolA derivatives are shown with their hexa-histidine extension. Additional amino acids added during the construction are indicated. Numbers correspond to the residue positions of wildtype TolA.

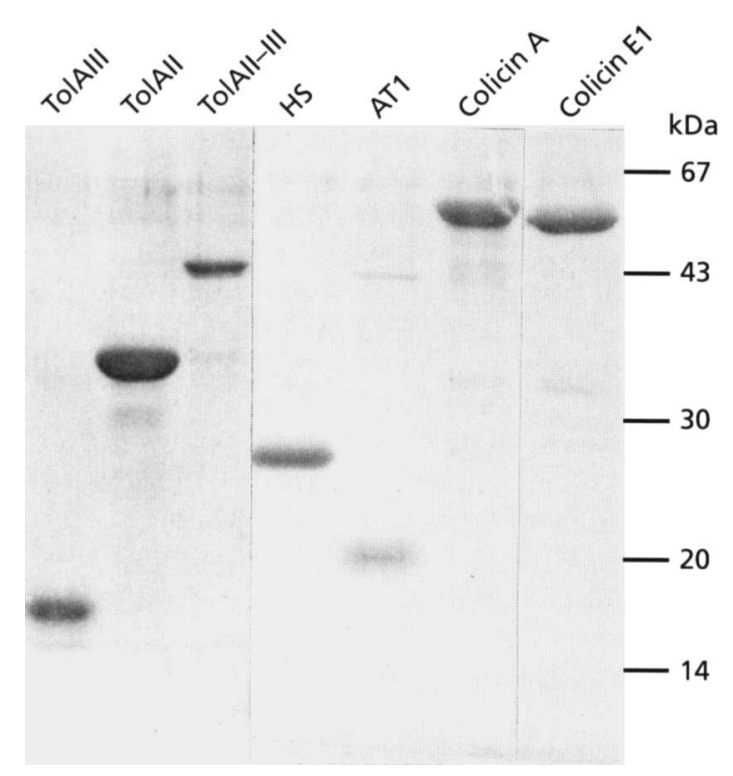

Fig. 2. Purified colicins and TolA domains used for the binding analyses. Coomassie blue-stained purified proteins used for surface plasmon experiments (the amounts of proteins ranging between 1 and $4 \mu \mathrm{g}$ ) were separated on $12.5 \%$ SDS-PAGE. on the surface of the sensor and the other (the analyte) is in solution. TolAII-III and TolAIII were immobilized on CM5 sensor chips through primary amino groups. Their interactions with colicins A, E1 and their $\mathrm{N}$ terminal domains were clearly detected, whereas no interaction was observed using colicin B, the C-terminal domain of colicin A, and the purified colicin A derivative deleted of residues 32 to 172 (Bénédetti et al., 1991) (not shown). BIAcore experiments clearly showed the interaction between HS or AT1 with TolAII-III and TolAIII domains, while no interaction occurred with TolAII. Fig. 4 shows the superimposition of two sensorgrams obtained with the $\mathrm{N}$-terminal domains of colicins A (AT1) and E1 (HS) injected at the same concentration onto immobilized TolAIII. Some differences between HS and AT1 on TolAIII were observed, with a stronger association and higher dissociation rate for HS. To characterize the colicin-TolA interaction, we also measured kinetic constants. Under normal experimental conditions, and with a homogeneous binding partner, the association is expected to be pseudo-first order, as the analyte is continuously replenished in the flow and so remains at a constant concentration during the injection. Concentrations of colicins (A, E1) and their N-terminal domains (AT1 and

Table 1. Western blot immunodetection of colicin binding to purified histidine-tagged TolA domains

Purified TolA derivatives were separated by Western blotting, then colicins or their $\mathrm{N}$-terminal domains interacting with TolA were immunodetected with the specified antibodies (polyclonal or monoclonal indicated by $\mathrm{pAb}$ or $\mathrm{mAb}$, respectively). For each test, immunodetection was first performed with the antibody alone to check that no background interaction occurred.

\begin{tabular}{|llccc|}
\hline & \multicolumn{1}{c}{ Antibody } & TolAII-III & TolAII & TolAIII \\
\hline Colicin A & mAb 1C11 & + & - & + \\
Colicin E1 & pAb HS & NS & Ns & + \\
Colicin E1 & pAb colicin E1 & + & - & + \\
Colicin B & pAb colicin B & - & - & - \\
AT1 & mAb 1C11 & + & - & + \\
HS & pAb HS & Ns & Ns & + \\
E1AA & mAb 3D6 & + & - & + \\
\hline
\end{tabular}

Ns, Signal was not specific because the antibody reacts weakly with the TolA domain. 


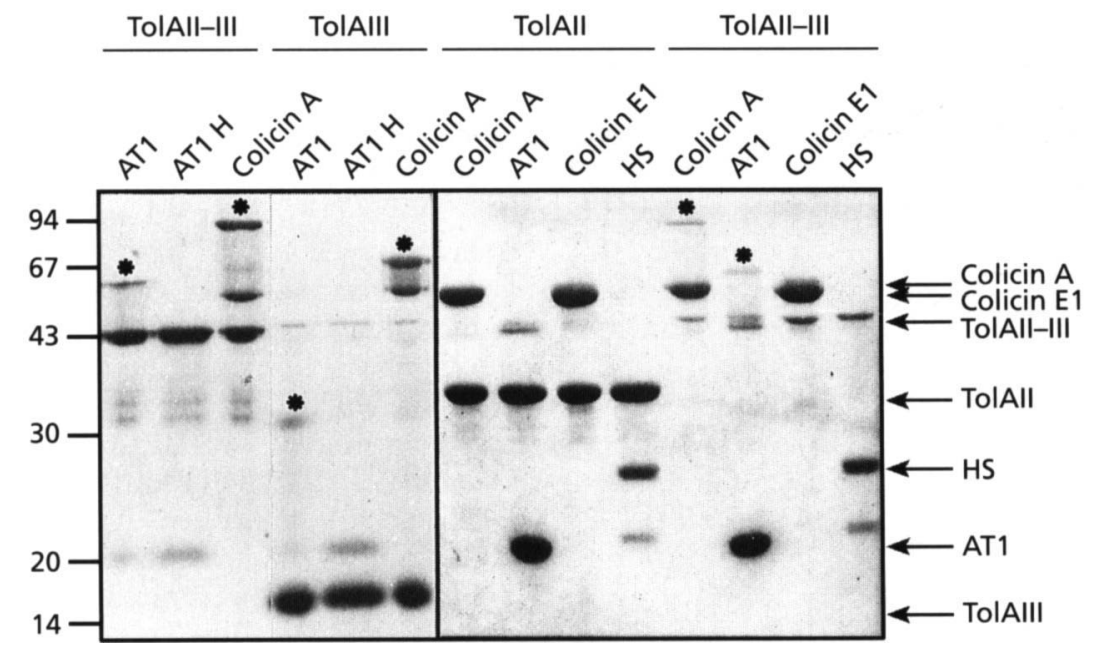

Fig. 3. Gel shift assay of colicin A and E1 and their $\mathrm{N}$-terminal domains interacting with TolA derivatives (stained with Coomassie blue). The samples were incubated for $20 \mathrm{~min}$ and heat-denatured where indicated $(\mathrm{H})$ prior to loading. TolA derivatives, colicins, $\mathrm{N}$-terminal domains (AT1 and HS) and complexes $\left(^{*}\right)$ are indicated.

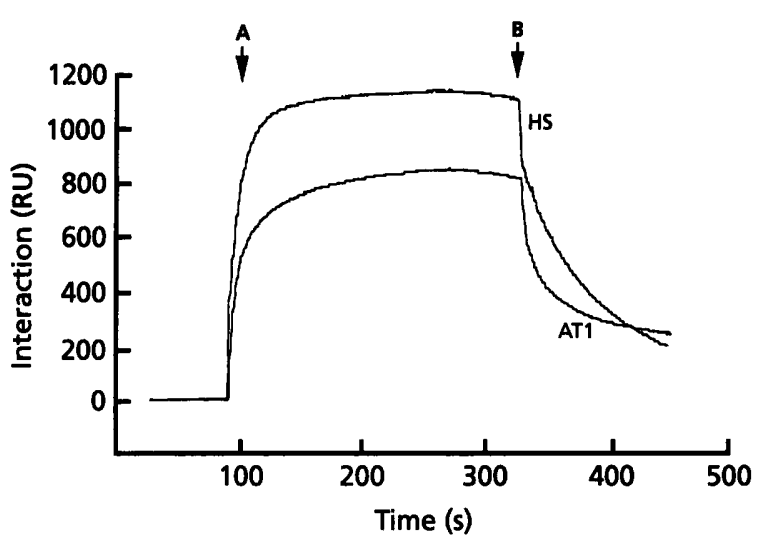

Fig. 4. Sensorgrams from the BIAcore showing the association and dissociation of AT1 and HS at the same concentration $(1.6 \mu \mathrm{M})$ on immobilized TolAlll. Arrow A indicates the beginning of protein injection, while arrow B corresponds to protein arrest in the flow buffer.

HS, respectively) were determined by an average of two amino acid compositions per protein. The homogeneity of each interactant was checked by SDS-PAGE (Fig. 2) and was at least $90 \%$. The analytical centrifugation experiments indicated the oligomeric state of the proteins. TolAIII and TolAII were monomeric in solution (data not shown), as was colicin A (Cavard et al., 1988) and its $\mathrm{N}$-terminal domain (Knibiehler et al., 1989). Two sets of four sensorgrams for each colicin fragment (concentrations ranging from 0.2 to $2.0 \mu \mathrm{M}$ ) were analysed using a pseudo-first-order kinetic model $(A+B \leftrightarrow A B)$. The experimental sensorgrams were poorly described by that homogeneous model, as indicated by the large magnitude of $\chi^{2}(>>10)$ associated with the best-fit description obtained by nonlinear regression analysis. Instead, a second exponential term in the integrated rate equation is required to describe the binding data more accurately, which involves a second, slower-binding phase. Apparent kinetic constants values were deduced using a more complex model: $A+B_{1}+B_{2} \leftrightarrow A B_{1}+A B_{2}$, where the response upon time is described by two exponential terms. In this case the quality of the fit was better, as indicated by the decreased magnitude of $\chi^{2}$ values (below 5 for HS and colicin E1 and generally below 10 for colicins A and AT1). Apparent values of $k_{\mathrm{a}}$ and $k_{\mathrm{d}}$ estimated for AT1 and HS on immobilized TolAIII and TolAII-III are shown in Table 2. The $K_{\mathrm{di}}$ was calculated as the ratio $k_{\mathrm{di}} / k_{\mathrm{ai}} \cdot K_{\mathrm{d} 1}$

Table 2. Apparent kinetic constants for AT1 and HS on immobilized TolAIII and TolAll-III

Data were obtained with about 1300 and 2000 RU of immobilized TolAIII and TolAII-III, respectively. Experiments were done at least twice. Equal amounts of complexes 1 and 2 were evaluated (from the model $A+B_{1}+B_{2} \leftrightarrow A B_{1}+A B_{2}$ ) except for HS-TolAIII, where complex 1 was predominant $(80 \%$ of complexes formed).

\begin{tabular}{|lllrrr|}
\hline Analyte & $\begin{array}{c}\text { Immobilized } \\
\text { protein }\end{array}$ & $\begin{array}{c}10^{-4} \times \boldsymbol{k}_{\mathrm{a} 1} \\
\left(\mathbf{M}^{-1} \mathbf{s}^{-1}\right)\end{array}$ & $\begin{array}{c}10^{-5} \times \boldsymbol{k}_{\mathrm{a} 2} \\
\left(\mathbf{M}^{-1} \mathbf{s}^{-1}\right)\end{array}$ & $\begin{array}{c}10^{3} \times \boldsymbol{k}_{\mathrm{d} 1} \\
\left(\mathbf{s}^{-1}\right)\end{array}$ & \multicolumn{1}{c|}{$\begin{array}{c}10^{2} \times \boldsymbol{k}_{\mathrm{d} 2} \\
\left(\mathbf{s}^{-1}\right)\end{array}$} \\
\hline AT1 & TolAIII & $1 \cdot 8 \pm 0 \cdot 4$ & $1 \cdot 2 \pm 0 \cdot 7$ & $4 \cdot 3 \pm 0 \cdot 2$ & $7 \cdot 2 \pm 0 \cdot 2$ \\
AT1 & TolAII-III & $3 \cdot 7 \pm 0 \cdot 6$ & $2 \cdot 8 \pm 1 \cdot 0$ & $6 \cdot 0 \pm 1 \cdot 8$ & $8 \cdot 1 \pm 0 \cdot 1$ \\
HS & TolAIII & $2 \cdot 8 \pm 2 \cdot 2$ & $10 \cdot 0 \pm 9 \cdot 5$ & $11 \cdot 0 \pm 2 \cdot 0$ & $3 \cdot 8 \pm 0 \cdot 1$ \\
HS & TolAII-III & $3 \cdot 0 \pm 2 \cdot 2$ & $1 \cdot 6 \pm 1 \cdot 0$ & $8 \cdot 6 \pm 1 \cdot 0$ & $2 \cdot 4 \pm 0 \cdot 3$ \\
\hline
\end{tabular}


and $K_{\mathrm{d} 2}$ values were estimated to be 0.2 and $0.6 \mu \mathrm{M}$ for AT1 and 0.4 and $0.04 \mu \mathrm{M}$ for HS, respectively. However, the $K_{\mathrm{d} 2}$ value obtained with HS was relevant for only $20 \%$ of complexes, while it represents $50 \%$ for AT1.

A putative TolA box formed with the pentapeptide DGSG(S/W) was suggested for the colicins E1, E2 and E3 as well as for colicin A (sequence DGTGW) (Pilsl \& Braun, 1995). We checked if such a peptide might compete with the interaction of the $\mathrm{N}$-terminal domain of colicin A and E1 (fixed concentration of $0.2 \mu \mathrm{M}$ ) with immobilized TolAIII. Two 11-mer peptides containing the specific pentapeptide and the surrounding sequence of colicin A and E1 were synthesized (see Methods). Using 1, 10, 100 and 1000 molar ratio of G11G peptide to HS protein, no decrease in the signal response was measured, compared to the results obtained in the absence of peptide. This result was the same when using an increasing ratio of G11E peptide with AT1 protein. According to the RU signal, which is related to the molecular mass of the interactant, we might expect a decrease in the RU signal if the peptide interacted with TolAIII. Our data indicated that no competition occurred with the two peptides containing the putative TolA boxes of colicins A and E1.

\section{DISCUSSION}

This study describes the purification of TolA soluble derivatives with six-histidine tagging extensions, and their interaction with the translocation domain of colicins A and E1. We have previously shown, using crude cell extracts and overlay immunodetections, that TolAIII interacts with colicin A and E1 and that this interaction might be relevant to the in vivo translocation of colicins (Bénédetti et al., 1991). Here, with purified proteins, different approaches were used to analyse these interactions in vitro.

The six-histidine tagging system and the tightly regulated arabinose promoter-operator vector were useful for the purification of TolA derivatives since initial attempts of TolA purification using the T7 expression system without any tagging yielded very low amounts of soluble TolA derivatives (not shown). The first step in affinity purification was performed by a batch procedure (using either $\mathrm{Nb}$ or $\mathrm{Cb}$ ), then a second step of purification by MonoS chromatography was necessary to remove contaminants. Using this approach, the arabinose isomerase was purified after elution with a low imidazole concentration. Optimization of production expression and the purification procedures is now in progress.

For the first time, we have demonstrated that the $\mathrm{N}$ terminal domain of colicin E1 (HS), like that of colicin A (AT1), can interact with the C-terminal domain of TolA (TolAIII). Overlay analyses were performed using various antibodies. Because we observed that the antibody directed against the N-terminal domain of colicin E1 (HS) cross-reacted with TolAII, we checked for the homology of HS against a protein database by using the
NCBI BLAST network service. A sequence of 123 residues within TolAII protein was found with $37 \%$ identity to that of HS (while no significant homology was found with AT1), which might explain the cross-reactivity of this antibody. The homologous regions corresponded to residue 43 to 156 and 113 to 226 of colicin E1 and TolA, respectively. The overlay assay using $\mathrm{HS}$ or the hybrid colicin E1AA followed by immunodetection using the anti-HS antibody (which does not cross-react with TolAIII) or the monoclonal antibody directed against colicin A demonstrated that TolAIII-HS interaction did occur. Mobility shift assays using SDS-PAGE confirmed the interaction of AT1 with TolAIII when the incubation was performed in the absence of SDS. According to the migration mobilities, the TolA-colicin complexes corresponded to dimers. However, by the same approach (SDS-PAGE followed by Coomassie blue staining), no complex was detected using colicin E1 or HS. This might indicate that the colicin E1-TolA interaction dissociated more rapidly during electrophoreses in the presence of SDS.

The last set of in vitro analyses was performed using SPR techniques. The first attempts at immobilization were obtained by amine coupling. Since the proteins contain numerous basic residues their coupling should generate some heterogeneity of presentation of the proteins on the chip. Another procedure using NTA coupling and further $\mathrm{Ni}^{2+}$-chelating (Gershon $\&$ Khilko, 1995) was performed but did not work for the TolAimmobilization step, although the histidine extension was still detected by Western blotting. This can probably be explained by a faster dissociation of the chelated TolA under SPR conditions. However, by the amine coupling approach, the qualitative results indicating that the N-terminal domains of colicin A and E1 specifically interacted with TolAIII were confirmed. We also checked that TolAIII interacted with immobilized colicins A and E1. Quantitative data obtained with the BIAcore enabled us to estimate only the apparent kinetic parameters, since sensorgram interpretations deviate from pseudo-first-order kinetics. Several factors are likely to cause this kind of deviation, such as heterogeneity of ligand sites within the matrix, restricted access of the analyte to the ligand sites because of mass transport limitation or parking problems resulting from possible masking of potential sites by bound analytes (O'Shannessy \& Winzor, 1996). The most common source, which was probably responsible in our experiments, is the heterogeneity of immobilized ligand sites, as the amine coupling chemistry used does not provide homogeneous presentation of the TolA molecules. Under these conditions, and knowing that the TolAIII protein was monomeric in solution and that the protein complex might correspond to a dimer (see above), the dissociation constants were found to be between 0.2 and $0.6 \mu \mathrm{M}$ for the N-terminal domains of colicins A and E1 binding to immobilized TolAIII. Elementary tests for self-consistency of kinetic analysis obtained with biosensor instruments have been recently proposed but they are inappropriate in this analysis as they assumed a 
simple binding model to be valid (Schuck \& Minton, 1996). However, due to the relative deviation values, no significant differences were found between TolAIII and TolAII-III. On the other hand, competition experiments using two peptides containing the putative TolA boxes located within the $\mathrm{N}$-terminal sequences of colicins $\mathrm{A}$ and E1 (Pilsl \& Braun, 1995) were checked in competition assays with their respective colicin. The peptide containing residues 8 to 19 of colicin A did not interfere with AT1 binding on TolAIII. This is in accordance with the result obtained by Bénédetti et al. (1991) showing that the colicin A derivative deleted of residues 32 to 172 did not interact with TolA in overlay experiments, and suggests that residues 8 to 19 of colicin A do not correspond to a TolA box. However, we did not find any competition with the peptide of colicin E1 on HS binding either. Because we do not have a positive control, such experiments using SPR will be useful to check for a peptide sequence inhibiting colicin interaction to give a clear conclusion. New experiments using the isothermal titration calorimetry (ITC) technique have been described to analyse the interactions between colicin $\mathrm{N}$, its $\mathrm{OmpF}$ receptor and other porins (Evans et al., 1996). Such experiments using the ITC or SPR techniques with colicins, their receptors and Tol proteins might provide useful thermodynamic and kinetic information about the translocation step of colicins through the outer membrane.

\section{ACKNOWLEDGEMENTS}

The authors thank Emmanuelle Bouveret for helpful discussion, Jean-Paul Briand for peptide syntheses, Nicole Zilber for amino acid compositions, Jacques Bonicel and Daniel Campese for $\mathrm{N}$-terminal protein sequences, Henri Bouteille and Paul Sauve for analytical centrifugation experiments and William A. Cramer for the gift of colicin E1. This work was supported by the Life Sciences department and the Mission Physique et Chimie du Vivant' of the CNRS.

\section{REFERENCES}

Baty, D., Knibiehler, M., Verheij, H., Pattus, F., Shire, D., Bernadac, A. \& Lazdunski, C. (1987). Site directed mutagenesis of the COOH-terminal region of colicin A: effect on secretion and voltage-dependent channel activity. Proc Natl Acad Sci USA 84, 1152-1156.

Bénédetti, H., Lazdunski, C. \& Lloubès, R. (1991). Protein import into Escherichia coli: colicins A and E1 interact with a component of their translocation system. EMBO J 10, 1989-1995.

Bouveret, E., Derouiche, R., Rigal, A., Lloubès, R., Lazdunski, C. \& Bénédetti, H. (1995). Peptidoglycan-associated lipoprotein-TolB interaction. J Biol Chem 270, 11071-11077.

Bouveret, E., Rigal, A., Lazdunski, C. \& Bénédetti, H. (1997). The N-terminal domain of colicin E3 interacts with TolB which is involved in the colicin translocation step. Mol Microbiol 23, 909-920.

Cagnon, C., Valverde, V. \& Masson, J. P. (1991). A new family of sugar-inducible expression vectors for Escherichia coli. Protein Eng 7, 843-847.

Cavard, D., Crozel, V., Gorvel, J.P., Pattus, F., Baty, D. \& Lazdunski, C. (1986). A molecular, genetic and immunological approach to the functioning of colicin $\mathrm{A}$, a pore-forming protein. J Mol Biol 187, 449-459.

Cavard, D., Sauve, P., Heitz, F., Pattus, F., Martinez, C., Dijkman, R. \& Lazdunski, C. (1988). Hydrodynamic properties of colicin A. Existence of a high-affinity lipid-binding site and oligomerization at acid pH. Eur J Biochem 172, 507-512.

Derouiche, R., Bénédetti, H., Lazzaroni, J. C., Lazdunski, C. \& Uoubes, R. (1995). Protein complex within Escherichia coli inner membrane - TolA N-terminal domain interacts with TolQ and TolR proteins. J Biol Chem 270, 11078-11084.

Derouiche, R., Gavioli, M., Bénédetti, H., Prilipov, A., Lazdunski, C. \& Loubès, R. (1996). TolA central domain interacts with Escherichia coli porins. EMBO J 15, 6408-6415.

Evans, L. J. A., Cooper, A. \& Lakey, J. H. (1996). Direct measurement of the association of a protein with a family of membrane receptors. J Mol Biol 255, 559-563.

Fognini-Lefebvre, N., Lazzaroni, J. C. \& Portalier, R. (1987). tolA, tolB and excC, three cistrons involved in the control of pleiotropic release of periplasmic proteins by Escherichia coli K12. Mol Gen Genet 209, 391-395.

Frenette, M., Bénédetti, H., Bernadac, A., Baty, D. \& Lazdunski, C. (1991). Construction, expression and release of hybrid colicins. $J$ Mol Biol 217, 421-428.

Gershon, P. D. \& Khilko, S. (1995). Stable chelating linkage for reversible immobilization of oligohistidine tagged proteins in the BIAcore surface plasmon resonance detector. JImmunol Methods $183,65-76$.

Guihard, G., Boulanger, P., Bénédetti, H., Lloubès, R., Besnard, M. \& Letellier, L. (1994). Colicin A and the Tol proteins involved in its translocation are preferentially located in the contact sites between the inner and outer membranes of Escherichia coli cells. $J$ Biol Chem 269, 5874-5880.

Isnard, M., Rigal, A., Lazzaroni, J. C., Lazdunski, C. \& Lloubès, R. (1994). Maturation and localization of the TolB protein required for colicin import. J Bacteriol 176, 6392-6396.

James, R., Kleanthous, C. \& Moore, G. R. (1996). The biology of E colicins: paradigms and paradoxes. Microbiology 142, 15691580.

Johnsson, B. \& Lofass, S. (1991). Immobilization of proteins to a carboxymethyldextran modified gold surface for biospecific interaction analysis in surface plasmon resonance. Anal Biochem 198, 268-277.

Kampfenkel, K. \& Braun, V. (1993). Membrane topologies of the TolQ and TolR proteins of Escherichia coli: inactivation of TolQ by a missense mutation in the proposed first transmembrane segment. J Bacteriol 175, 4485-4491.

Knibiehler, M., Howard, S.P., Baty, D., Géli, V., Lloubès, R. \& Lazdunski, C. (1989). Isolation and molecular and functional properties of the amino-terminal domain of colicin A. Eur J Biochem 181, 109-113.

Lazdunski, C. (1995). Colicin import and pore formation: a system for studying protein transport across membrane. Mol Microbiol 16, 1059-1066.

Lazzaroni, J. C. \& Portalier, R. (1992). The excC gene of Escherichia coli $\mathrm{K}-12$ required for cell envelope integrity encodes the peptidoglycan-associated lipoprotein (PAL). Mol Microbiol 6, 735-742.

Lazzaroni, J. C., Vianney, A., Popot, J. L., Benedetti, H., Samatey, F., Lazdunski, C., Portalier, R. \& Geli, V. (1995). Transmembrane alpha-helix interactions are required for the functional assembly of the Escherichia coli Tol complex. J Mol Biol 246, 1-7.

Levengood, S. K. \& Webster, R. E. (1989). Nucleotide sequences of 
the tolA and tolB genes and localization of their products, components of a multistep translocation system in $E$. coli. J Bacteriol 171, 6600-6609.

Levengood, S. K., Beyer, W. F. \& Webster, R. E. (1991). TolA: a membrane protein involved in colicin uptake contains an extended helical region. Proc Natl Acad Sci USA 88, 5939-5943.

Levengood-Freyermuth, S. K., Click, E. M. \& Webster, R. E. (1993). Role of the carboxyterminal domain of TolA in protein import and the integrity of the outer membrane. J Bacteriol 175, 222-228.

Muller, M. M., Vianney, A., Lazzaroni, J. C., Webster, R. E. \& Portalier, R. (1993). Membrane topology of the Escherichia coli TolR protein required for cell envelope integrity. $J$ Bacteriol 175, 6059-6061.

O'Shannessy, D. J. \& Winzor, G. (1996). Interpretation of deviations from pseudo-first-order kinetic behavior in the characterization of ligand binding by biosensor technology. Anal Biochem 236, 275-283.

O'Shannessy, D. J., Brigham Burke, M., Soneson, K. K., Hensley, P. \& Brooks, I. (1993). Determination of rate and equilibrium constants for macromolecular interactions using surface plasmon resonance: use of nonlinear least square analysis methods. Anal Biochem 212, 457-468.

Perez-Perez, J. \& Gutierrez, J. (1995). An arabinose-inducible expression vector, pAR3, compatible with ColE1-derived plasmids. Gene 158, 141-142.

Pilsl, H. \& Braun, V. (1995). Novel colicin 10: assignment of four domains to TonB- and TolC-dependent uptake via the Tsx receptor and to pore formation. Mol Microbiol 16, 57-67.

Postle, K. (1993). TonB protein and energy transduction between membranes. J Bioenerg Biomembr 25, 591-601.

Schuck, P. \& Minton, A. P. (1996). Kinetic analysis of biosensor data: elementary tests for self-consistency. Trends Biochem Sci 21, 458-460.

Sun, T. P. \& Webster, R. E. (1986). fii, a bacterial locus required for filamentous phage infection and its relation to colicin-tolerant tolA and tolB. $J$ Bacteriol 165, 107-115.

Vianney, A., Lewin, T. M., Beyer, W. F., Jr, Lazzaroni, J. C., Portalier, R. \& Webster, R. E. (1994). Membrane topology and mutational analysis of the TolQ protein of $E$. coli required for the uptake of macromolecules and cell envelope integrity. J Bacteriol 176, 822-829.

Vianney, A., Muller, M. M., Clavel, T., Lazzaroni, J. C., Portalier, R. \& Webster, R. E. (1996). Characterization of the tol-pal region of Escherichia coli K-12: translational control of tolR expression by TolQ and identification of a new open reading frame downstream of pal encoding a periplasmic protein. J Bacteriol 178, 4031-4038.

Webster, R. E. (1991). The tol gene products and the import of macromolecules into Escherichia coli. Mol Microbiol 5, 10051011.

Received 7 March 1997; revised 12 June 1997; accepted 23 June 1997. 